\title{
Relay and complete synchronization in heterogeneous multiplex networks of chaotic maps
}

Cite as: Chaos 30, 061104 (2020); doi: 10.1063/5.0008902

Submitted: 26 March 2020 . Accepted: 21 May 2020 .

Published Online: 9 June 2020

E. Rybalova, ,a) (D) G. Strelkova, , ${ }^{1, b)}$ (i) E. Schöll, ${ }^{2, c)}$ (D) and V. Anishchenko ${ }^{1, d)}$ (D)

\begin{abstract}
AFFILIATIONS
${ }^{1}$ Department of Physics, Saratov State University, 83 Astrakhanskaya Street, Saratov 410012, Russia

${ }^{2}$ Institut für Theoretische Physik, Technische Universität Berlin, Hardenbergstr. 36, 10623 Berlin, Germany
\end{abstract}

a) Electronic mail: rybalovaev@gmail.com

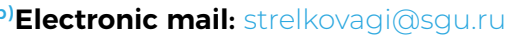

${ }^{c}$ Electronic mail: schoell@physik.tu-berlin.de

d) Author to whom correspondence should be addressed: wadim@sgu.ru

\begin{abstract}
We study relay and complete synchronization in a heterogeneous triplex network of discrete-time chaotic oscillators. A relay layer and two outer layers, which are not directly coupled but interact via the relay layer, represent rings of nonlocally coupled two-dimensional maps. We consider for the first time the case when the spatiotemporal dynamics of the relay layer is completely different from that of the outer layers. Two different configurations of the triplex network are explored: when the relay layer consists of Lozi maps while the outer layers are given by Henon maps and vice versa. Phase and amplitude chimera states are observed in the uncoupled Henon map ring, while solitary state regimes are typical for the isolated Lozi map ring. We show for the first time relay synchronization of amplitude and phase chimeras, a solitary state chimera, and solitary state regimes in the outer layers. We reveal regimes of complete synchronization for the chimera structures and solitary state modes in all the three layers. We also analyze how the synchronization effects depend on the spatiotemporal dynamics of the relay layer and construct phase diagrams in the parameter plane of inter-layer vs intra-layer coupling strength of the relay layer.
\end{abstract}

Published under license by AIP Publishing. https://doi.org/10.1063/5.0008902

Synchronization in complex networks of coupled oscillators is one of the most important phenomena of self-organization and can be observed in natural, social, and technological systems. ${ }^{1-8}$ Recently, special attention has been given to the collective dynamics and synchronization effects in multilayer and multiplex networks, ${ }^{9-11}$ in which layers can exhibit different spatiotemporal structures such as chimera states $^{12-15}$ or solitary states. ${ }^{16,17}$ Remote or relay synchronization ${ }^{18}$ represents an important feature allowing distant control and coordination, which is particularly valuable in neurophysiology ${ }^{19-21}$ and communication technology. ${ }^{22}$ This form of synchronization occurs between two distant (outer) layers that are not directly coupled but mediated by a relay (remote) layer. Very recently, relay synchronization has attracted much interest, which is particularly related to the discovery of chimera states, ${ }^{12,13}$ where a network spontaneously splits into coexisting spatially localized clusters of elements with incoherent and coherent dynamics, respectively. Remote synchronization has been explored in multiplex networks of continuousand discrete-time systems, ${ }^{15,18,23,24}$ and for different types of intra-layer architecture, e.g., scale-free or Erdös-Renyi, ${ }^{18}$ and random topologies of small-world type. ${ }^{25}$ However, in these cases, all layers consist of identical nodes with the same type of local dynamics. In the present work, we study for the first time relay and complete synchronization in a heterogeneous three-layer network of chaotic maps. The system under study includes an intermediate (relay) ensemble (layer) and two remote ensembles (outer layers) that are symmetrically coupled with the relay layer. All the three layers are represented by rings of nonlocally coupled discrete-time oscillators but the individual dynamics of the relay layer elements is completely different from that of the outer layers. We consider two different configurations: when the individual elements of the central layer and of the outer layers are 
described by Lozi maps and Henon maps, respectively, and vice versa. This enables one to observe various types of spatiotemporal structures, such as solitary states in the Lozi map ring, ${ }^{26-28}$ amplitude, and phase chimeras in the Henon map ring, ${ }^{26-29}$ when the subnetworks are uncoupled. In this work, we show for the first time relay and complete synchronization of these structures and a solitary state chimera, ${ }^{30,31}$ in a heterogeneous multiplex network of discrete maps. We also analyze the role of the relay layer structure in forming synchronous patterns and observing relay and complete synchronization.

\section{INTRODUCTION}

Over many years, collective dynamics of complex ensembles of interacting subsystems has been a topic of special attention of experts in different scientific fields. ${ }^{1,32-38}$ This is related to the fact that many phenomena in living and inanimate nature occur due to the interaction between a large number of individual elements that are coupled with each other in different ways. Of most interest are complex networks of nonlinear elements that exhibit rich dynamics and various self-organization effects. A prominent example is synchronization phenomena. Synchronization is one of the most important nonlinear effects in nature and technology. ${ }^{1-8}$ Synchronization phenomena take place both in selfsustained oscillatory systems (synchronization of limit cycle, ${ }^{69,70}$ chaos synchronization ${ }^{6,71,72}$ ) and in application to spatiotemporal structures in complex ensembles. ${ }^{1,4,7,8}$ Over the past two decades, an important insight into the synchronization phenomena has been related to the discovery of a so-called chimera state, ${ }^{12,13}$ which represents an intriguing example of partial spatial synchronization. The chimera is a state where spatially localized coherent (synchronous) and incoherent (asynchronous) clusters of oscillators coexist in the network. ${ }^{12,13,39}$ Chimera states were first discovered in ensembles of nonlocally coupled identical Kuramoto oscillators. ${ }^{12,39}$ Afterward, they were found in networks of oscillatory systems with periodic and chaotic dynamics, ${ }^{26-29,40-50}$ in neural networks, ${ }^{51-55}$ and also for global and local coupling ${ }^{56-60}$ between the elements. Chimeras were also observed in a number of experiments. ${ }^{61-68}$

Nowadays, the attention of researchers is focused on the effects that can occur not only in isolated (individual) ensembles but in large systems of interacting ensembles. The interaction between ensembles (layers) in multilayer networks can lead to different types of synchronization of complex spatiotemporal structures, including chimeras, e.g., cluster synchronization, ${ }^{73,74}$ complete synchronization, ${ }^{75}$ generalized synchronization, ${ }^{76}$ phase synchronization, ${ }^{77}$ external and mutual synchronization, ${ }^{78-80}$ explosive synchronization, ${ }^{81-83}$ etc.

Recently, relay or remote synchronization has received special attention of researchers. This form of synchronization takes place between two distant nodes (elements) of a network, which are not directly coupled but interact via an intermediate (relay) node. Remote synchronization was first reported as one of the specific peculiarities of star-like networks. A star-like structure includes a single hub node connected with several peripheral ones that form star rays. Nodes which belong to different rays are not connected with each other. The formation of synchronized clusters in such star-like networks was considered in a number of papers. ${ }^{84-86}$ Remote (relay) synchronization occurs in networks of nonidentical self-sustained oscillators as phase synchronization of peripheral nodes when the hub is not synchronized with them. This type of synchronization was studied for starlike networks of periodic oscillators, ${ }^{87}$ chaotic oscillators, ${ }^{88,89}$ van der Pol oscillators, ${ }^{90}$ and also for scale-free networks. ${ }^{9}$

Remote synchronization has also been detected experimentally in laser systems ${ }^{88}$ and circuits of chaotic oscillators. ${ }^{18,87,92}$ This type of synchronization has an outstanding importance for exploring the information transmission in brain networks. ${ }^{19-21}$ Recent studies have been addressed to relay synchronization in multiplex or multilayer networks, ${ }^{15,18,22-24,93}$ where the individual layers can represent ensembles of continuous- or discrete-time systems and exhibit complex spatiotemporal structures, including chimera states. ${ }^{15,23,24}$ Besides, it has been shown that relay synchronization occurs in multiplex networks with different types of intra-layer topology such as scale-free or Erdös-Renyi, ${ }^{18}$ and random inhomogeneities of smallworld type ${ }^{25}$ However, in all cases, both relay and remote layers are represented by ensembles of the same type of oscillators.

The purpose of the present work is to study relay and complete synchronization in a heterogeneous three-layer network of discrete-time oscillators where the relay layer is completely different in its spatiotemporal dynamics from that of the remote layers. In our study, each layer is represented by a ring of nonlocally coupled chaotic maps. As individual elements, we choose the Henon map ${ }^{96}$ and the Lozi map. ${ }^{94}$ We explore two different configurations of the triplex network: when the relay layer is described by a Lozi map ring, while the outer layers are given by Henon map rings, and vice versa. When the rings are uncoupled, the Henon map ensemble demonstrates phase and amplitude chimera states, ${ }^{26-29}$ and the Lozi map ring typically exhibits solitary states. ${ }^{26-28}$ We show for the first time relay and complete synchronization of amplitude and phase chimeras, a solitary state chimera, ${ }^{30,31}$ and solitary state regimes in heterogeneous triplex networks of chaotic maps. We also analyze how these synchronization effects depend on the spatiotemporal structure in the relay layer and construct maps of synchronization regimes in the parameter plane of inter-layer vs intra-layer coupling strength of the relay layer.

\section{MODEL}

We study the dynamics of a multiplex network that consists of three pairwise coupled subnetworks and is schematically pictured in Fig. 1. Each layer is represented by a ring of nonlocally coupled discrete-time oscillators (maps) in a chaotic regime. The considered triplex network is heterogeneous, i.e., the middle ring includes elements that are completely different in their individual dynamics from those of the outer layers that are in turn identical.

As individual elements we choose classical two-dimensional chaotic discrete-time systems, the Lozi map, ${ }^{94}$

$$
\begin{aligned}
& x^{L}(n+1)=f_{x}\left(x^{L}(n), y^{L}(n)\right)=1-\alpha\left|x^{L}(n)\right|+y^{L}(n), \\
& y^{L}(n+1)=f_{y}\left(x^{L}(n), y^{L}(n)\right)=\beta x^{L}(n),
\end{aligned}
$$




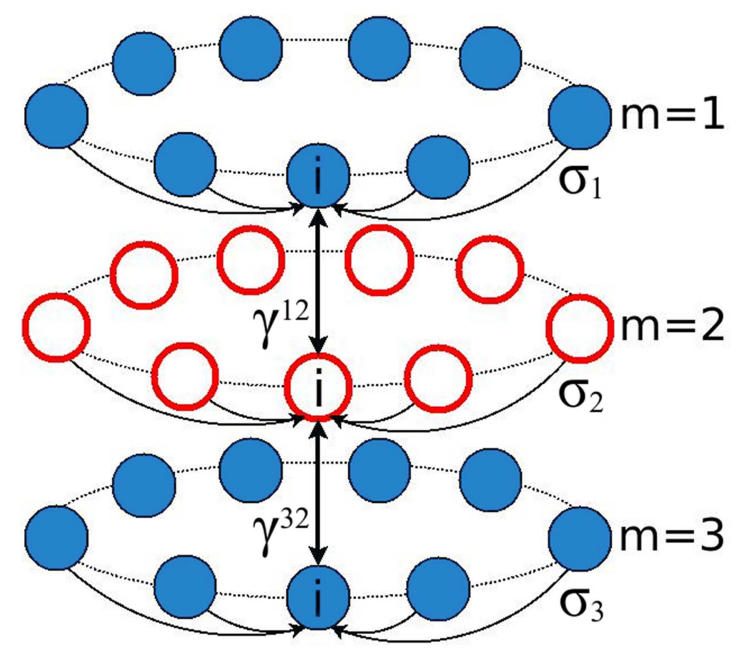

FIG. 1. The scheme of the three-layer multiplex network given by system (3). The index $m$ denotes the layer number, and the intra-layer coupling is given by $\sigma_{m}$. The outer layers $m=1$ and $m=3$ are pairwise and symmetrically coupled with the middle layer $m=2$ with the inter-layer coupling $\gamma^{12}$ and $\gamma^{32}$, respectively.

and the Henon map, ${ }^{71,96}$

$$
\begin{aligned}
& x^{H}(n+1)=f_{x}\left(x^{H}(n), y^{H}(n)\right)=1-\alpha\left(x^{H}(n)\right)^{2}+y^{H}(n), \\
& y^{H}(n+1)=f_{y}\left(x^{H}(n), y^{H}(n)\right)=\beta x^{H}(n) .
\end{aligned}
$$

In both maps, $x^{L, H}, y^{L, H}$ are dynamical variables, $n$ denotes the discrete time. The control parameters $\alpha$ and $\beta$ are fixed as $\alpha=1.4$ and $\beta=0.3$, which correspond to chaotic dynamics in both individual uncoupled maps.

The individual Lozi map (1) is known to be characterized by a quasihyperbolic chaotic attractor. ${ }^{95}$ As was shown in Refs. 26-28, the ring of nonlocally coupled Lozi maps demonstrates the transition from complete coherence to incoherence through the appearance of solitary states ${ }^{16}$ when the coupling strength between the elements decreases.

The isolated Henon map (2) represents a classical example of a discrete-time system with a nonhyperbolic chaotic attractor. ${ }^{6,96,97}$ When Henon maps are nonlocally coupled within a ring, the transition from complete chaotic synchronization to spatiotemporal chaos is accompanied by the emergence of amplitude and phase chimera states with decreasing coupling strength. ${ }^{26-28,46}$

The multiplex network schematically drawn in Fig. 1 is described by the following system of equations:

$$
\begin{aligned}
x_{i}^{m}(n+1)= & f_{x}\left(x_{i}^{m}(n), y_{i}^{m}(n)\right)+\frac{\sigma_{m}}{2 R_{m}} \sum_{j=i-R_{m}}^{i+R_{m}}\left[f_{x}\left(x_{j}^{m}(n), y_{j}^{m}(n)\right)\right. \\
& \left.-f_{x}\left(x_{i}^{m}(n), y_{i}^{m}(n)\right)\right]+\sum_{l=1}^{3} \gamma^{m l} G_{i}^{m l}(n), \\
y_{i}^{m}(n+1)= & f_{y}\left(x_{i}^{m}(n), y_{i}^{m}(n)\right),
\end{aligned}
$$
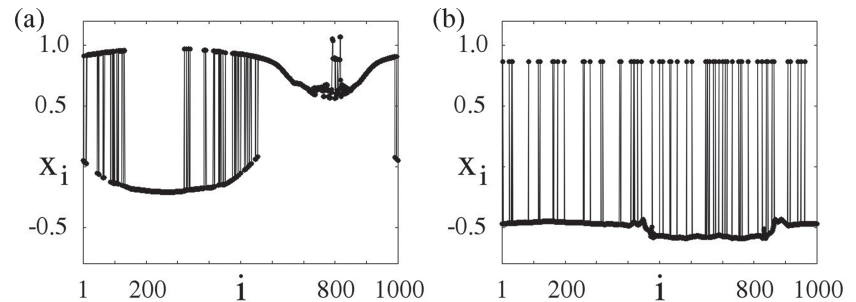

FIG. 2. Examples of initial spatial distributions (snapshots) of the $x$ values in the Henon map ring (a) and the Lozi map ring (b) in the uncoupled outer layers $(\gamma=0)$ in network (3). Parameters: $\alpha=1.4, \beta=0.3, R=320, N=1000$, and $\sigma_{1}=\sigma_{3}=0.31(\mathrm{a})$ and $\sigma_{1}=\sigma_{3}=0.18(\mathrm{~b})$.

where $m=1,2,3$ is the layer number, $i=1,2, \ldots, N_{m}$ is the node number in each layer, and $N_{m}=N=1000$ is the total number of elements in each layer. Functions $f_{x}$ and $f_{y}$ are defined by the righthand parts of maps (1) and (2).

The layers in network (3) are pairwise and symmetrically coupled in a triplex configuration. The inter-layer coupling is introduced via the $x$ variables and described by the third term in (3). $\gamma^{m l}$ characterizes the inter-layer coupling strength between the individual elements of the corresponding layers $m$ and $l . G_{i}^{m l}(n)$ represents the inter-layer coupling function which is of dissipative nature and is given as follows:

$$
G_{i}^{m l}(n)=f_{x}\left(x_{i}^{l}(n), y_{i}^{l}(n)\right)-f_{x}\left(x_{i}^{m}(n), y_{i}^{m}(n)\right) .
$$

By analogy with the work, ${ }^{24}$ we represent the inter-layer coupling strength in the matrix form,

$$
\gamma^{m l}=\left(\begin{array}{ccc}
0 & \gamma^{12} & 0 \\
\gamma^{21} & 0 & \gamma^{23} \\
0 & \gamma^{32} & 0
\end{array}\right),
$$

where the row and column numbers are related to the corresponding layers. Thus, each element of matrix (5) describes the coupling between the corresponding layers. In our numerical simulation, the inter-layer coupling is chosen to be identical, $\gamma^{21}=\gamma^{12}=\gamma^{23}$ $=\gamma^{32}=\gamma$.

The nonlocal intra-layer coupling in the $m$ th layer is given by the coupling strength $\sigma_{m}$ and the coupling range $R_{m}$, i.e., the number of neighbors to which the $i$ th element is coupled from each side within the corresponding ring. The intra-layer coupling is given by the second term in (3). In our numerics, the intra-coupling range is fixed the same for all the layers, $R_{m}=R=320$.

Before introducing the inter-laying coupling, the individual uncoupled rings are iterated for the period of $n=4 \times 10^{4}$ time units, which is treated as a transient process to achieve a steady-state regime in each layer. The initial conditions for the $x$ and $y$ variables of the Lozi map ring are randomly distributed within the range $[-0.5 ; 0.5]$. For the Henon map ring, the initial $x$ and $y$ values are randomly chosen from the intervals $[-0.5 ; 0.5]$ and $[-0.15 ; 0.15]$, respectively.

We consider two different situations: when the middle (relay) layer is given by the Lozi map ring and the outer layers are described by the Henon map rings [the Henon-Lozi-Henon (HLH) network], 
and vice versa [the Lozi-Henon-Lozi (LHL) network]. When the layers are uncoupled, chimera states are observed in the Henon map ring and a solitary state regime in the Lozi map ring. Figure 2 exemplifies typical steady-state spatiotemporal structures that are established in the isolated Henon and Lozi rings when they are chosen as outer layers, before the inter-layer coupling is switched on. In this case, $\sigma_{1}=\sigma_{3}=0.31$ for the Henon map rings and $\sigma_{1}=\sigma_{3}$ $=0.18$ for the Lozi map rings. As seen from Fig. 2(a), for the chosen values of the intra-layer coupling, a combined chimera structure is formed in the Henon map ring and consists of incoherence clusters of the phase chimera $(1 \leqslant i \leqslant 133,320 \leqslant i \leqslant 524$, and $988 \leqslant i$ $\leqslant 1000)$ and of the amplitude chimera $(720 \leqslant i \leqslant 850)$. The Lozi map ring demonstrates a solitary state mode [Fig. 2(b)].

Since the ensembles in network (3) demonstrate complex spatiotemporal structures of different types (amplitude and phase chimeras, solitary states) when the layers are uncoupled, it is useful to quantify the degree of synchronization of these structures between the layers. For this purpose, we use the local time-averaged variance of the instantaneous values of the $x$ variables for each node $i$ of the corresponding layers,

$$
\begin{aligned}
\delta_{i}^{m l} & =\frac{1}{T} \sum_{n=1}^{T}\left(x_{i}^{m}(n)-x_{i}^{l}(n)\right)^{2}, \\
i & =1,2, \ldots, N .
\end{aligned}
$$

The synchronization effect (in its global sense) between two layers in the triplex network (3) is measured using the variance of the amplitudes, averaged over time and ensemble elements. This quantity is defined as follows:

$$
\delta^{m l}=\frac{1}{N} \sum_{i=1}^{N}\left(\frac{1}{T} \sum_{n=1}^{T}\left(x_{i}^{m}(n)-x_{i}^{l}(n)\right)^{2}\right),
$$

where the time-averaging is carried out over $T=10^{4}$ iterations after the transient process. This measure quantifies the global degree of synchronization between the layers; it is zero for perfect synchronization, and non-zero for desynchronization. It can be used to evaluate the regime of synchronization (relay and complete) and desynchronization when the inter-layer coupling $\gamma$ is varied.

Taking into account the fact that we deal with a heterogeneous network, synchronization effects can be treated only in their effective sense, i.e., a synchronization criterion must be specified. We assume that layers $m$ and $l$ are synchronized if the following condition is valid:

$$
\delta^{m l}<0.005
$$

This value is arbitrary, and any sufficiently small value of $\delta^{m l}$ may be chosen as a threshold, but the results remain qualitatively the same. If $\delta^{13}<0.005, \delta^{12}>0.005$ and $\delta^{23}>0.005$, then the effective relay synchronization appears in network (3). The effective complete synchronization is observed in the system provided that $\delta^{13}<0.005$, $\delta^{12}<0.005$, and $\delta^{23}<0.005$. The same conditions are applied to the local measure (6).

\section{SYNCHRONIZATION IN THE HENON-LOZI-HENON NETWORK}

We first consider the triplex network (3) when the intermediate (relay) layer is represented by the Lozi map ensemble, while the outer layers are given by the Henon map rings. We fix the intra-layer coupling in the layers as follows: $\sigma_{1}=\sigma_{3}=0.31$ and $\sigma_{2}=0.14$. When the layers are uncoupled, the Henon map rings demonstrate amplitude and phase chimeras as exemplified in Fig. 2(a), and the Lozi map ring shows an incoherent solitary state regime.

We vary the inter-layer coupling $\gamma$ and calculate the global variance $\delta^{m l}$ according to Eq. (7) between the layers. The corresponding dependences of $\delta^{m l}$ vs $\gamma$ are plotted in Fig. 3 in a logarithmic scale and marked by different symbols for the corresponding pairs of subnetworks. The inset shows a subrange in a linear scale. Using these plots and applying the synchronization criterion (8), we can define which kind of synchronization occurs in the network (3). When the inter-layer coupling is sufficiently weak $(\gamma<0.14)$, $\delta^{m l}>0.005$. This implies that the network (3) is desynchronized. At $\gamma=0.14$, the value of the global variance between the first and the third layer $\delta^{13}<0.005$ and thus satisfies the synchronization condition (8). At the same time, $\delta^{12}$ and $\delta^{23}>0.005$. This result indicates the effective relay synchronization in the network (3). With increasing $\gamma>0.14$ up to $\gamma=0.6, \delta^{13}=0$ (they are shown in the inset in Fig. 3), and thus we can conclude that full relay synchronization is confidently detected and observed in the network (3). As is clearly seen from the plot in Fig. 3, the measures $\delta^{12}$ and $\delta^{23}$ take small but nonzero values $(0.01)$ within the interval of the inter-layer coupling $\gamma$ variation $[0.13,0.32]$ and even grow for $\gamma \geqslant 0.32$. Thus, the global variance between the first and the second layer and the second and the third layer does not satisfy the synchronization condition (8). Therefore, one can conclude that complete synchronization does not occur in the Henon-Lozi-Henon network.

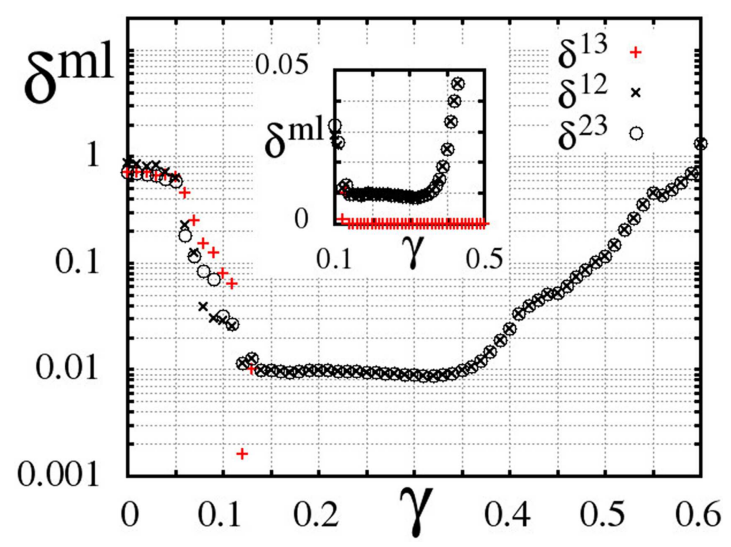

FIG. 3. Dependences of the global synchronization between the first and the third layer measured by the variance $\delta^{13}$ (red crosses), between the first and the second layer $\delta^{12}$ (black crosses), and between the second and the third layer $\delta^{23}$ (black circles) in the Henon-Lozi-Henon network vs the inter-layer coupling $\gamma$ plotted in a logarithmic scale. The inset shows the range of relay synchronization in a linear scale. Parameters: $\sigma_{1}=\sigma_{3}=0.31, \sigma_{2}=0.14, R=320, \alpha=1.4, \beta=0.3$, and $N=1000$ 
Figure 4 shows snapshots of the dynamics of each layer (a)-(c) and the local variance (6) between the first and the third layer $\delta_{i}^{13}$ (d) and between the first and the second layer $\delta_{i}^{12}$ (e) for each node $i$ for three selected values of the inter-layer coupling $\gamma$ (panels I, II, and III) in the Henon-Lozi-Henon network. The results presented in the first (I) panel in Fig. 4 correspond to a small value of the interlayer coupling $\gamma=0.07$. As can be seen from Figs. 4,I(d) and 4,I(e), all the elements of the network in the incoherent domains remain practically desynchronized. Due to the interaction, the spatiotemporal regimes in the layers influence each other and start changing significantly. The solitary states in the middle (relay) layer seem to "push" certain elements of the first and third layers out of the coherent mode [Figs. 4,I(a) and 4,I(c)]. The chimera states formed in the outer layers distort the coherent parts in the relay layer and phase chimeras are induced in the second layer [Fig. 4,I(b)]. Nevertheless, one can observe that the synchronization condition (8) is satisfied for the coherent parts of the outer layers $\delta_{i}^{13}<0.005$ [Fig. 4,I(d)]. In this case, one can speak about partial relay synchronization as first described in Ref. 23.

When the inter-layer coupling becomes stronger, $\gamma=0.23$, full relay synchronization takes place in the network as clearly depicted in the second (II) panel in Fig. 4. The spatiotemporal structures in the first and third layers are identical [Figs. 4,II(a) and 4,II(c)] and the local synchronization measure $\delta_{i}^{13} \simeq 0$ [Fig. 4,II(d)] satisfies criterion (8). The influence of the relay layer structure, which initially consists of solitary states, leads to the formation of solitary state clusters in the outer layers. Such a structure represents a solitary state chimera first reported in Refs. 30 and 31. In Figs. 4,II(a) and $4, \mathrm{II}(\mathrm{c})$ the incoherence cluster of the solitary state chimera occupies the regions $20 \leqslant i \leqslant 120$ and $920 \leqslant i \leqslant 990$ and coexists with the phase chimera $(320 \leqslant i \leqslant 415$ and $880 \leqslant i \leqslant 900)$ and coherence regions. Thus, we demonstrate relay synchronization of the solitary state chimera for the Henon-Lozi-Henon network for the first time. It is also worth noting that the interaction between the layers induces the appearance of a completely new structure in the relay layer (in the Lozi map ring), a solitary state chimera [Fig. 4,II(b)], which has never been observed in this ensemble without inter-layer coupling. Although the spatial structure in the relay layer [Fig. 4,II(b)] qualitatively repeats the modes in the outer layers, the global variances $\delta^{12}=\delta^{23}=0.009642$ (Fig. 3) do no satisfy the synchronization condition (8). Thus, in this case and for these parameter values, complete synchronization does not occur in the considered network. Note that it is the solitary state chimera that is least synchronized with the relay layer [Fig. 4,II(e)].

A further increase of the inter-layer coupling, $\gamma=0.48$, leads to the destruction of the chimera structures and transition (a)

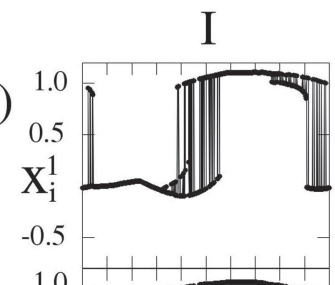

(b)

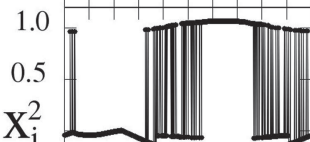

(c)

$-0.5$

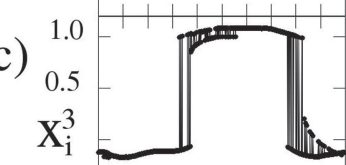

(d)

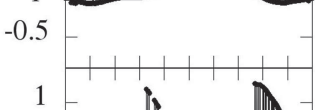

(e)
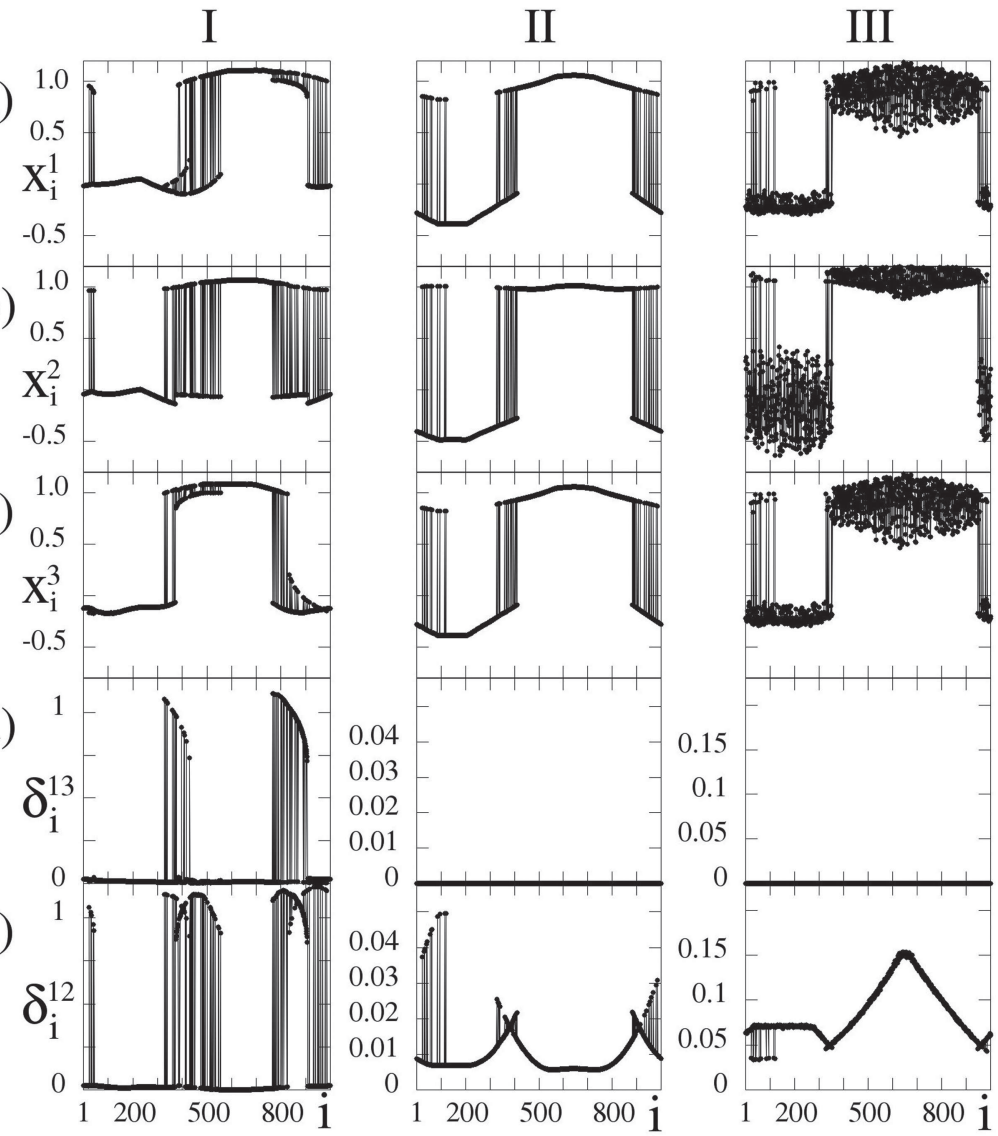

FIG 4. Dynamics of the rings in the Henon-Lozi-Henon network for different values of the inter-layer coupling $\gamma: 0.07$ (I panel), 0.23 (II panel), and 0.48 (III panel). (a)-(c) Snapshots of $x_{i}^{1}, x_{i}^{2}, x_{i}^{3}$, local variance (6) between the first and the third layer $\delta_{i}^{13}(\mathrm{~d})$, and between the first and the second layer $\delta_{i}^{12}(\mathrm{e})$ vs the element number $i$. Other parameters are as in Fig. 3 . 
to completely incoherence regimes in all the three layers [Figs. 4,III(a)-4,III(c)]. Nevertheless, similarly to the previous case (Fig. 4,II), the skeleton of these modes remains chimera-like and is defined by the initial chimera structures in the outer layers. The phase chimera clusters become narrow, while the incoherence clusters of the amplitude chimera occupy the most part of the ring elements $(1 \leqslant i \leqslant 325$ and $355 \leqslant i \leqslant 945)$. Such regimes can be observed within a narrow range of strong inter-layer coupling. Full relay synchronization is well pronounced, $\delta_{i}^{13} \simeq 0$ [Fig. 4,III(d)], while $\delta_{i}^{12}$ and $\delta_{i}^{23}$ have sufficiently large values [Fig. 4,III(e)].

The changes in the spatiotemporal structures in the network layers described above are observed in the entire range of the intralayer coupling in the relay layer $\left(\sigma_{2} \in[0 ; 0.5]\right)$ and when the interlayer coupling increases. These modifications can be summarized in the following sequence: emergence of a solitary state chimera and relay synchronization of the phase and solitary state chimeras $\rightarrow$ appearance of an amplitude chimera and relay synchronization of the phase and amplitude chimeras $\rightarrow$ expanding of the amplitude chimera and relay synchronization of the phase and amplitude chimeras $\rightarrow$ relay synchronization of incoherence regimes. In addition, it is important to note that the structures in the outer layers (the Henon map rings in the considered case) have a dominant effect in the change of spatiotemporal regimes in all the three layers as the inter-layer coupling strength increases.

We now analyze how the Henon-Lozi-Henon network dynamics depends on the intra-layer coupling $\sigma_{2}$ of the relay layer with increasing inter-layer coupling $\gamma$. The isolated Lozi map ring can demonstrate coherent spatiotemporal regimes and solitary state regimes as $\sigma_{2}$ decreases. ${ }^{27,28}$ For the chosen parameter values of the Lozi map ring ( $\alpha=1.4, \beta=0.3$, and $R=320$ ), a solitary state regime is observed within the interval $[0 ; 0.23]$.

Synchronization and desynchronization regions for the HenonLozi-Henon network are shown in the $\left(\gamma, \sigma_{2}\right)$ parameter plane in Fig. 5. The layers are desynchronized in the white region, complete synchronization is observed inside the red-hatched region 1, relay synchronization occurs within the black-hatched region 2 , and trajectories diverge to infinity in the yellow region 3. As can be seen from Fig. 5, complete synchronization is achieved within a bounded range for the intra-layer coupling of the relay layer $\sigma_{2} \in[0.22 ; 0.48]$ and characterized by threshold values and finite ranges for the interlayer coupling depending on $\sigma_{2}$ values. The value of $\sigma_{2}=0.22$ in the isolated Lozi map ring is close to the boundary of transition from solitary state regimes to coherence regimes with increasing $\sigma_{2}$. Relay synchronization (region 2 in Fig. 5) is observed within a finite interval of the inter-layer coupling $\gamma \in[0.11,0.65]$ and takes place for any value of the intra-layer coupling $\sigma_{2}$ in the relay layer. The latter means that relay synchronization takes place independently of which dynamical regime occurs in the relay layer. Moreover, the boundary values of the relay synchronization region in the interlayer coupling do not practically change and only slightly fluctuate depending on $\sigma_{2}$. Our calculations and Fig. 5 indicate that the emergence of solitary states and an increase of their number with decreasing intra-layer coupling $\sigma_{2}<0.22$ prevents complete synchronization in the Henon-Lozi-Henon network but does not affect relay synchronization of the outer layers. In our case, complete synchronization occurs when coherence regimes are observed in the relay layer.

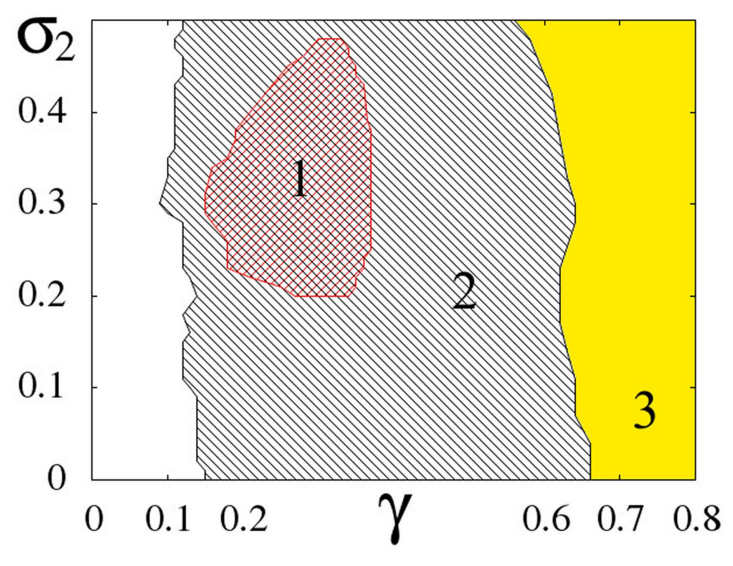

FIG. 5. Synchronization and desynchronization regions for the Henon-Lozi-Henon network in the $\left(\gamma, \sigma_{2}\right)$ parameter plane. The white region corresponds to desynchronized layers, red-hatched region 1 corresponds to complete synchronization $\left(\delta^{\mathrm{ml}}>0.005\right)$, black-hatched region 2 relates to relay synchronization $\left(\delta^{13}<0.005, \delta^{23}>0.005, \delta^{13}>0.005\right)$, and trajectories diverge to infinity in yellow region 3 . Other parameters are as in Fig. 3. Sampling step-size in $\sigma_{2}$ and $\gamma$ is 0.01 . Averaging over three sets of random initial conditions is used.

\section{SYNCHRONIZATION EFFECTS IN THE LOZI-HENON-LOZI NETWORK}

We now discuss the dynamics of the triplex network (3) in the case when the relay layer is represented by the Henon map ring and the outer layers by the Lozi map rings. When the subnetworks are uncoupled, the Lozi map rings show solitary state regimes as depicted in Fig. 2(b), and the Henon map ring demonstrates a combined chimera structure at $\sigma_{2}=0.13$.

As in the previous case, we vary the inter-layer coupling strength $\gamma$ and calculate the global synchronization measure $\delta^{m l}(7)$ for each pair of the subnetworks. The numerical results are presented in Fig. 6 in a logarithmic scale. The inset again shows a subrange in a linear scale. As can be seen from the dependence $\delta^{13}(\gamma)$ (red crosses in Fig. 6), the outer layers begin to synchronize even for very weak coupling $(\gamma \simeq 0.10)$. The value of $\delta^{13}$ is precisely zero within the range $\gamma \in[0.10,0.58]$, as can be seen in the inset in Fig. 6 . Full relay synchronization $\left(\delta^{13} \simeq 0\right)$ appears already at $\gamma \simeq 0.1$, which is less than in the case of the Henon-Lozi-Henon network (Fig. 3). Besides, in this case, the effective complete synchronization $\left(\delta^{13}<0.005, \delta^{23}<0.005\right.$, and $\left.\delta^{13}<0.005\right)$ is clearly observed when the inter-layer coupling $\gamma$ is varied within the interval $[0.21,0.38]$. A further increase in the inter-layer coupling leads to the loss of complete synchronization, while relay synchronization persists up to $\gamma=0.58$.

The peculiarities of the Lozi-Henon-Lozi network dynamics are illustrated in Fig. 7 by exemplary snapshots (a)-(c) and the local synchronization measures $\delta_{i}^{13}$ (d) and $\delta_{i}^{12}$ (e) for three different values of the inter-layer coupling $\gamma$ (panels I, II, and III). The first (I) panel in Figs. 7,I(a)-7,I(e) shows numerical results for the case of a very weak coupling between the layers $\gamma=0.05$. It is seen that all the layers are desynchronized. Increasing $\gamma$ [Figs. 7,II(a)-7,II(e) 


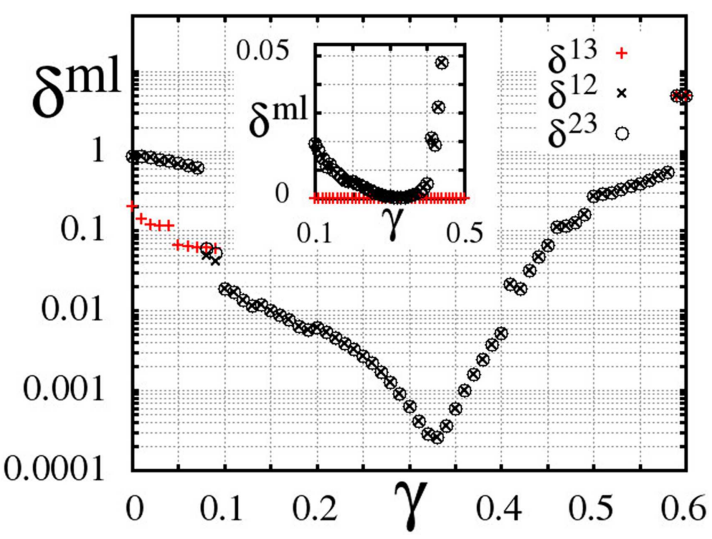

FIG. 6. Synchronization between the first and the third layer measured by the variance $\delta^{13}$ (red crosses), between the first and the second layer $\delta^{12}$ (black crosses), and between the second and the third layer $\delta^{23}$ (black circles) in the Lozi-Henon-Lozi network vs the inter-layer coupling $\gamma$ shown in a logarithmic scale. The inset shows the range of relay synchronization in a linear scale. Parameters: $\sigma_{1}=\sigma_{3}=0.18, \sigma_{2}=0.13, R=320, \alpha=1.4, \beta=0.3$, and $N=1000$ for $\gamma=0.17$ leads to a serious modification of the spatiotemporal structures in all the three layers and the formation of an amplitude chimera structure (incoherence clusters include nodes $1 \leqslant i$ $\leqslant 120,350 \leqslant i \leqslant 650$, and $880 \leqslant i \leqslant 1000$ ) coexisting with a finite number of solitary states [Figs. 7,II(a)-7,II(c)]. Besides, as follows from Fig. 7,II(d), full relay synchronization $\delta_{i}^{13} \simeq 0$ of such a complex structure is clearly observed in the network, while there is no complete synchronization, $\delta_{i}^{12}>0.005$ [Fig. 7,II(e)].

When the inter-layer coupling becomes stronger, e.g., $\gamma=0.36$ (Fig. 7,III), the number of solitary states in all the three layers significantly increases and the chimera-like structure is completely destroyed [Figs. 7,III(a)-7,III(c)]. As in the previous case, the dynamical regimes in the outer layers play a defining role in forming spatiotemporal structures in the whole network with increasing inter-layer coupling. As seen from Figs. 7,III(a)-7,III(e), both relay and complete synchronization of the incoherence solitary state regime are achieved in the Lozi-Henon-Lozi network. Complete synchronization takes place since $\delta_{i}^{12}$ is less than 0.005 but not zero [Fig. 7,III(e)]. Note that in both networks, the oscillators in the solitary state regime are characterized by larger values of the local variance (6) as compared with the other network elements.

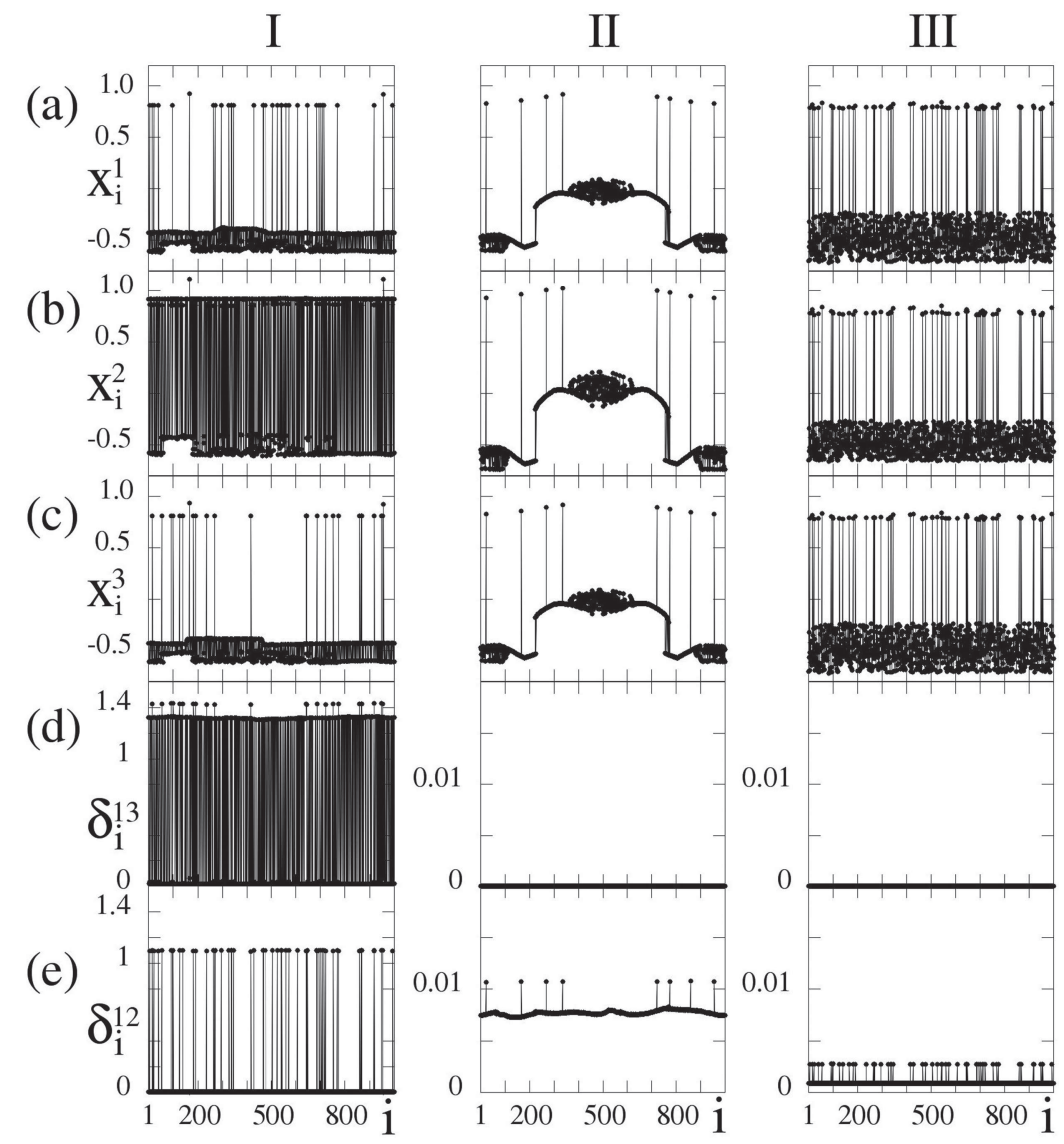

FIG. 7. Dynamics of the rings in the Lozi-Henon-Lozi network for different values of the inter-layer coupling $\gamma: 0.05(I)$, 0.17 (II), and 0.36 (III). (a)-(c) Snapshots of $x_{i}^{1}, x_{i}^{2}, x_{i}^{3}$, local variance (6) between the first and the third layer $\delta_{i}^{13}(\mathrm{~d})$, and between the first and the second layer $\delta_{i}^{12}(\mathrm{e})$ vs node index $i$. Other parameters are as in Fig. 6. 


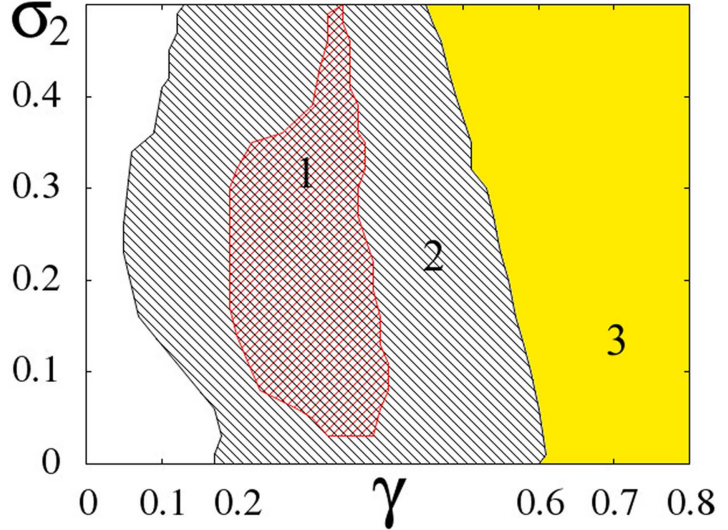

FIG. 8. Synchronization and desynchronization regions for the Lozi-Henon-Lozi network in the $\left(\gamma, \sigma_{2}\right)$ parameter plane. The notations are the same as in Fig. 5. Other parameters are as in Fig. 6.

Now we vary the intra-layer coupling in the relay layer $\left(\sigma_{2}\right)$ and construct regions of synchronization and desynchronization in the Lozi-Henon-Lozi network when the inter-layer coupling $\gamma$ increases. Results of the simulations are shown in the twodimensional diagram in Fig. 8, where the same notations are used as in Fig. 5. As is seen from Fig. 8, relay synchronization is observed in the Lozi-Henon-Lozi network within the whole range of intra-layer coupling $\sigma_{2} \in[0,0.5]$. However, as compared with the previous case (Fig. 5), both left and right boundaries of the inter-layer coupling $\gamma$ range are shifted toward smaller values and depend on $\sigma_{2}$. For example, relay synchronization appears already at a very small coupling strength $\gamma \simeq 0.05$ when $\sigma_{2}$ is varied within the interval $[0.17,0.35]$. Note that amplitude and phase chimera states are typically formed within that range in the individual uncoupled Henon map ring. Complete synchronization takes place in the Lozi-Henon-Lozi network for almost the whole range of the intralayer coupling $\sigma_{2}$ (Fig. 8) starting with its very small value when the elements in the relay layer are almost desynchronized. Unlike the previous case (Fig. 5), the complete synchronization region for the Lozi-Henon-Lozi network is larger in the inter-layer coupling strength but its width strongly depends on $\sigma_{2}$. The widest ranges of $\gamma$ correspond to those values of $\sigma_{2}$ when chimera structures are observed in the isolated Henon map ring.

\section{CONCLUSION}

We have demonstrated effects of relay and complete synchronization in a heterogeneous multiplex network of three coupled ensembles (layers) of chaotic oscillators. Each layer is represented by a one-dimensional ring of nonlocally coupled two-dimensional chaotic discrete-time systems. As individual elements, the Henon and Lozi maps have been used. The individual Henon map ring exhibits amplitude and phase chimera structures and the uncoupled Lozi map ring typically demonstrates solitary state regimes. The two outer layers in the triplex network have not been directly coupled but interact via the intermediate (relay) layer. We have considered two different cases, namely, when the relay layer is described by the Henon map ring, while the outer layers are given by the Lozi map rings, and vice versa. We have shown for the first time the possibility of achieving relay and complete synchronization of the amplitude and solitary state chimera as well as solitary state regimes. We have also analyzed how the heterogeneous triplex network dynamics depends both on the inter-layer coupling $\gamma$ and the intra-layer coupling strength of the relay layer $\sigma_{2}$ and have defined the relay and complete synchronization regimes in the $\left(\gamma, \sigma_{2}\right)$ parameter plane. The numerical results described in this paper can be treated as a generalization of the findings obtained earlier for a three-layer multiplex network of nonlocally coupled logistic maps ${ }^{24}$ to the case of a heterogeneous triplex network in which the outer and relay layers consist of chaotic discrete-time oscillators with different individual dynamics.

\section{ACKNOWLEDGMENTS}

This work was supported by the Deutsche Forschungsgemeinschaft (DFG, German Research Foundation)-Projektnummer 163436311-SFB 910 and by the RFBR and the DFG according to the Research Project No. 20-52-12004.

\section{DATA AVAILABILITY}

The data that support the findings of this study are available within the article and its supplementary material.

\section{REFERENCES}

${ }^{1}$ V. I. Nekorkin and M. G. Velarde, Synergetic Phenomena in Active Lattices (Springer, Berlin, 2002).

${ }^{2}$ A. Pikovsky, M. Rosenblum, and J. Kurths, Synchronization. A Universal Concept in Nonlinear Sciences (Cambridge University Press, Cambridge, 2001).

${ }^{3}$ E. Mosekilde, Y. Maistrenko, and D. Postnov, Chaotic Synchronization: Applications to Living Systems (World Scientific, Singapore, 2002).

${ }^{4}$ G. Osipov, J. Kurths, and C. Zhou, Synchronization in Oscillator Networks (Springer, Berlin, 2007).

${ }^{5}$ A. G. Balanov, N. B. Janson, D. E. Postnov, and O. V. Sosnovtseva, Synchronization: From Simple to Complex (Springer, Berlin, 2009).

${ }^{6}$ V. S. Anishchenko, T. E. Vadivasova, and G. I. Strelkova, Deterministic Nonlinear Systems. A Short Course, Springer Series in Synergetics (Springer, Berlin, 2014).

${ }^{7}$ E. Schöll, S. H. L. Klapp, and P. Hövel, Control of Self-organizing Nonlinear Systems (Springer, Berlin, 2016).

${ }^{8}$ S. Boccaletti, A. N. Pisarchik, C. I. del Genio, and A. Amann, Synchronization: From Coupled Systems to Complex Networks (Cambridge University Press, Cambridge, 2018).

${ }^{9}$ M. De Domenico, A. Sole-Ribalta, E. Cozzo, M. Kivelä, Y. Moreno, M. A. Porter, S. Gómez, and A. Arenas, "Mathematical formulation of multilayer networks," Phys. Rev. X 3, 041022 (2013).

${ }^{10}$ S. Boccaletti, G. Bianconi, R. Criado, C. I. del Genio, J. Gomez-Gardeñes, M. Romance, I. Sendiña-Nadal, Z. Wang, and M. Zanin, "The structure and dynamics of multilayer networks,” Phys. Rep. 544, 1122 (2014).

${ }^{11}$ M. Kivelä, A. Arenas, M. Barthelemy, J. P. Gleeson, Y. Moreno, and M. A. Porter, “Multilayer networks," J. Complex Netw. 2, 203-271 (2014).

${ }^{12}$ Y. Kuramoto and D. Battogtokh, "Coexistence of coherence and incoherence in nonlocally coupled phase oscillators," Nonlin. Phen. Complex Sys. 5(4), 380-385 (2002).

${ }^{13}$ D. M. Abrams and S. H. Strogatz, "Chimera states for coupled oscillators," Phys. Rev. Lett. 93(17), 174102 (2004). 
${ }^{14}$ S. Ghosh, A. Zakharova, and S. Jalan, "Non-identical multiplexing promotes chimera states," Chaos Soliton. Fract. 106, 56-60 (2018).

${ }^{15}$ J. Sawicki, S. Ghosh, S. Jalan, and A. Zakharova, "Chimeras in multiplex networks: Interplay of inter- and intra-layer delays,” Front. Appl. Math. Stat. 5, 19 (2019).

${ }^{16}$ Y. Maistrenko, B. Penkovsky, and M. Rosenblum, "Solitary state at the edge of synchrony in ensembles with attractive and repulsive interactions," Phys. Rev. E 89, 060901 (2014).

${ }^{17}$ M. Mikhaylenko, L. Ramlow, S. Jalan, and A. Zakharova, "Weak multiplexing in neural networks: Switching between chimera and solitary states," Chaos 29, 023122 (2019).

${ }^{18}$ I. Leyva, I. Sendiña-Nadal, R. Sevilla-Escoboza, V. P. Vera-Avila, P. Chholak, and S. Boccaletti, "Relay synchronization in multiplex networks," Sci. Rep. 8, 8629 (2018).

${ }^{19}$ R. Guillery and S. M. Sherman, "Thalamic relay functions and their role in corticocortical communication: Generalizations from the visual system," Neuron 33, 163-175 (2002).

${ }^{20}$ A. S. Mitchell, S. M. Sherman, M. A. Sommer, R. G. Mair, R. P. Vertes, and Y. Chudasama, "Advances in understanding mechanisms of thalamic relays in cognition and behavior," J. Neurosci. 34(46), 15340-15346 (2014).

${ }^{21} \mathrm{~V}$. Vlasov and A. Bifone, "Hub-driven remote synchronization in brain networks,” Sci. Rep. 7, 10403 (2017).

${ }^{22}$ L. Zhang, A. E. Motter, and T. Nishikawa, "Incoherence-mediated remote synchronization," Phys. Rev. Lett. 118(17), 174102 (2017).

${ }^{23}$ J. Sawicki, I. Omelchenko, A. Zakharova, and E. Schöll, "Delay controls chimera relay synchronization in multiplex networks," Phys. Rev. E 98, 062224 (2018).

${ }^{24}$ M. Winkler, J. Sawicki, I. Omelchenko, A. Zakharova, V. Anishchenko, and E. Schöll, "Relay synchronization in multiplex networks of discrete maps," Europhys. Lett. 126, 50004 (2019).

${ }^{25}$ F. Drauschke, J. Sawicki, R. Berner, I. Omelchenko, and E. Schöll, "Effect of topology upon relay synchronization in triplex neuronal networks," Chaos 30, 051104 (2020).

${ }^{26}$ N. Semenova, A. Zakharova, E. Schöll, and V. S. Anishchenko, "Does hyperbolicity impede emergence of chimera states in networks of nonlocally coupled chaotic oscillators?," Europhys. Lett. 112, 40002 (2015).

${ }^{27}$ E. Rybalova, N. Semenova, G. Strelkova, and V. Anishchenko, "Transition from complete synchronization to spatiotemporal chaos in coupled chaotic systems with nonhyperbolic and hyperbolic attractors," Eur. Phys. J. Sp. Top. 226(9), 1857-1866 (2017).

${ }^{28}$ N. I. Semenova, E. V. Rybalova, G. I. Strelkova, and V. S. Anishchenko, "Coherence-incoherence transition in ensembles of nonlocally coupled chaotic oscillators with nonhyperbolic and hyperbolic attractors," Regul. Chaotic Dyn. 22(2), 148-162 (2017).

${ }^{29}$ I. Omelchenko, B. Riemenschneider, P. Hövel, Y. Maistrenko, and E. Schöll, "Transition from spatial coherence to incoherence in coupled chaotic systems," Phys. Rev. E 85, 026212 (2012).

${ }^{30}$ A. Bukh, E. Rybalova, N. Semenova, G. Strelkova, and V. Anishchenko, "New type of chimera and mutual synchronization of spatiotemporal structures in two coupled ensembles of nonlocally interacting chaotic maps," Chaos 27(11), 111102 (2017).

${ }^{31}$ E. V. Rybalova, G. I. Strelkova, and V. S. Anishchenko, "Mechanism of realizing a solitary state chimera in a ring of nonlocally coupled chaotic maps," Chaos Soliton. Fract. 115, 300-305 (2018).

${ }^{32}$ G. Nicolis and I. Prigogine, Self-Organization in Nonequilibrium Systems (Wiley-Interscience, New York, 1977).

${ }^{33}$ H. Haken, Synergetics (Springer, Berlin, 1977).

${ }^{34}$ Y. Kuramoto, Chemical Oscillations, Waves and Turbulence (Springer, Berlin, 1984).

${ }^{35}$ V. S. Afraimovich, V. I. Nekorkin, G. V. Osipov, and V. D. Shalfeev, Stability, Structures and Chaos in Nonlinear Synchronization Networks (World Scientific, Singapore, 1995).

${ }^{36}$ I. R. Epstein and J. A. Pojman, An Introduction to Nonlinear Chemical Dynamics: Oscillations, Waves, Patterns, and Chaos (Oxford University Press, Oxford, 1998).

${ }^{37}$ A. T. Winfree, The Geometry of Biological Time (Springer, Berlin, 2001).
${ }^{38}$ H. Malchow, S. V. Petrovskii, and E. Venturino, Spatiotemporal Patterns in Ecology and Epidemiology: Theory, Models, and Simulation (Chapman and Hall, London, 2007).

${ }^{39}$ M. J. Panaggio and D. M. Abrams, "Chimera states: Coexistence of coherence and incoherence in networks of coupled oscillators," Nonlinearity 28(3), R67 (2015).

${ }^{40}$ A. Zakharova, M. Kapeller, and E. Schöll, "Chimera death: Symmetry breaking in dynamical networks,” Phys. Rev. Lett. 112(15), 154101 (2014).

${ }^{41} \mathrm{E}$. Schöll, "Synchronization patterns and chimera states in complex networks: Interplay of topology and dynamics," Eur. Phys. J. Spec. Top. 225, 891 (2016).

${ }^{42}$ O. E. Omel'chenko, Y. Maistrenko, and P. Tass, "Chimera states: The natural link between coherence and incoherence,” Phys. Rev. Lett. 100(4), 044105 (2008). ${ }^{43}$ A. Zakharova, Chimera Patterns in Complex Networks (Springer, Berlin, 2020). ${ }^{44}$ I. Omelchenko, Y. Maistrenko, P. Hövel, and E. Schöll, "Loss of coherence in dynamical networks: Spatial chaos and chimera states," Phys. Rev. Lett. 106(23), 234102 (2011).

${ }^{45}$ D. Dudkowski, Y. Maistrenko, and T. Kapitaniak, "Different types of chimera states: An interplay between spatial and dynamical chaos," Phys. Rev. E 90, 032920 (2014).

${ }^{46}$ S. A. Bogomolov, A. V. Slepnev, G. I. Strelkova, E. Schöll, and V. S. Anishchenko, "Mechanisms of appearance of amplitude and phase chimera states in ensembles of nonlocally coupled chaotic systems," Commun. Nonlinear Sci. Numer. Simul. 43, 25-36 (2017).

${ }^{47}$ A. V. Slepnev, A. V. Bukh, and T. E. Vadivasova, "Stationary and non-stationary chimeras in an ensemble of chaotic self-sustained oscillators with inertial nonlinearity," Nonlinear Dyn. 88(4), 2983-2992 (2017).

${ }^{48}$ I. A. Shepelev, A. V. Bukh, G. I. Strelkova, T. E. Vadivasova, and V. S. Anishchenko, "Chimera states in ensembles of bistable elements with regular and chaotic dynamics," Nonlinear Dyn. 90, 2317 (2017).

${ }^{49}$ A. zur Bonsen, I. Omelchenko, A. Zakharova, and E. Schöll, "Chimera states in networks of logistic maps with hierarchical connectivities," Eur. Phys. J. B 91, 65 (2018).

${ }^{50}$ A. K. Malchow, I. Omelchenko, E. Schöll, and P. Hövel, "Robustness of chimera states in nonlocally coupled networks of nonidentical logistic maps," Phys. Rev. E 98, 012217 (2018).

${ }^{51}$ I. Omelchenko, E. Omel'chenko, P. Hövel, and E. Schöll, "When nonlocal coupling between oscillators becomes stronger: Patched synchrony or multichimera states," Phys. Rev. Lett. 110(22), 224101 (2013).

${ }^{52}$ I. Omelchenko, A. Provata, J. Hizanidis, E. Schöll, and P. Hövel, "Robustness of chimera states for coupled FitzHugh-Nagumo oscillators," Phys. Rev. E 91, 022917 (2015).

${ }^{53}$ N. Semenova, A. Zakharova, V. S. Anishchenko, and E. Schöll, "Coherenceresonance chimeras in a network of excitable elements," Phys. Rev. Lett. 117, 014102 (2016).

${ }^{54}$ A. Schmidt, T. Kasimatis, J. Hizanidis, A. Provata, and P. Hövel, "Chimera patterns in two-dimensional networks of coupled neurons," Phys. Rev. E 95(3), 032224 (2017).

${ }^{55}$ I. A. Shepelev, T. E. Vadivasova, A. V. Bukh, G. I. Strelkova, and V. S. Anishchenko, "New type of chimera structures in a ring of bistable FitzHugh-Nagumo oscillators with nonlocal interaction," Phys. Lett. A 381(16), 1398-1404 (2017).

${ }^{56}$ A. Yeldesbay, A. Pikovsky, and M. Rosenblum, "Chimeralike states in an ensemble of globally coupled oscillators,” Phys. Rev. Lett. 112, 144103 (2014).

${ }^{57}$ L. Schmidt and K. Krischer, "Clustering as a prerequisite for chimera states in globally coupled systems,” Phys. Rev. Lett. 114, 034101 (2015).

${ }^{58}$ F. Böhm, A. Zakharova, E. Schöll, and K. Lüdge, "Amplitude-phase coupling drives chimera states in globally coupled laser networks," Phys. Rev. E 91, 040901 (2015).

${ }^{59}$ C. R. Laing, “Chimeras in networks with purely local coupling," Phys. Rev. E 92, 050904(R) (2015).

${ }^{60}$ S. Kundu, S. Majhi, B. K. Bera, D. Ghosh, and M. Lakshmanan, "Chimera states in two-dimensional networks of locally coupled oscillators," Phys. Rev. E 97, 022201 (2015).

${ }^{61}$ A. M. Hagerstrom, T. E. Murphy, R. Roy, P. Hövel, I. Omelchenko, and E. Schöll, "Experimental observation of chimeras in coupled-map lattices," Nat. Phys. 8, 658-661 (2012). 
${ }^{62} \mathrm{M}$. R. Tinsley, S. Nkomo, and K. Showalter, "Chimera and phase cluster states in populations of coupled chemical oscillators," Nat. Phys. 8, 662-665 (2012).

${ }^{63} \mathrm{M}$. Wickramasinghe and I. Z. Kiss, "Spatially organized dynamical states in chemical oscillator networks: Synchronization, dynamical differentiation, and chimera patterns," PLoS One 8(11), e80586 (2013).

${ }^{64}$ L. V. Gambuzza, A. Buscarino, S. Chessari, L. Fortuna, R. Meucci, and M. Frasca, "Experimental investigation of chimera states with quiescent and synchronous domains in coupled electronic oscillators," Phys. Rev. E 90, 032905 (2014).

${ }^{65}$ T. Kapitaniak, P. Kuzma, J. Wojewoda, K. Czolczynski, and Y. Maistrenko, "Imperfect chimera states for coupled pendula," Sci. Rep. 4, 6379 (2014).

${ }^{66} \mathrm{~L}$. Larger, B. Penkovsky, and Y. Maistrenko, "Laser chimeras as a paradigm for multistable patterns in complex systems," Nat. Commun. 6, 7752 (2015).

${ }^{67}$ V. I. Ponomarenko, D. D. Kulminskiy, and M. D. Prokhorov, "Chimeralike states in networks of bistable time-delayed feedback oscillators coupled via the mean field," Phys. Rev. E 96, 022209 (2017).

${ }^{68}$ J. F. Totz, J. Rode, M. R. Tinsley, K. Showalter, and H. Engel, "Spiral wave chimera states in large populations of coupled chemical oscillators," Nat. Phys. 14, 282-285 (2017).

${ }^{69}$ A. A. Andronov, E. A. Vitt, and S. E. Khaikin, Theory of Oscillations (Pergamon Press, Oxford, 1966).

${ }^{70} \mathrm{I}$. Blekhman, Synchronization in Science and Technology (ASME, New York, 1988).

${ }^{71}$ V. S. Anishchenko, V. V. Astakhov, A. B. Neiman, T. E. Vadivasova, and L. Schimansky-Geier, Nonlinear Dynamics of Chaotic and Stochastic Systems: Tutorial and Modern Developments, 1st ed. (Springer, Berlin, 2000).

${ }^{72}$ V. S. Anishchenko, T. E. Vadivasova, D. E. Postnov, and M. A. Safonova, "Synchronization of chaos," Int. J. Bifurc. Chaos 2, 633 (1992).

${ }^{73}$ L. M. Pecora, F. Sorrentino, A. M. Hagerstrom, T. E. Murphy, and R. Roy, "Cluster synchronization and isolated desynchronization in complex networks with symmetries," Nat. Commun. 5, 4079 (2014).

${ }^{74} \mathrm{~S}$. Jalan and A. Singh, "Cluster synchronization in multiplex networks," Europhys. Lett. 113(3), 30002 (2016).

${ }^{75}$ C. I. del Genio, J. Gómez-Gardeñes, J. I. Bonamassa, and S. Boccaletti, "Synchronization in networks with multiple interaction layers," Sci. Adv. 2, e1601679 (2016).

${ }^{76}$ R. G. Andrzejak, G. Ruzzene, and I. Malvestio, "Generalized synchronization between chimera states," Chaos 27, 053114 (2017).

${ }^{77}$ R. G. Andrzejak, G. Ruzzene, I. Malvestio, K. Schindler, E. Schöll, and A. Zakharova, "Mean field phase synchronization between chimera states," Chaos 28(9), 091101 (2018).

${ }^{78}$ A. V. Bukh, G. I. Strelkova, and V. S. Anishchenko, "Synchronization of chimera states in coupled networks of nonlinear chaotic oscillators," Russ. J. Nonlinear Dyn. 14(4), 419-433 (2018)

${ }^{79}$ G. I. Strelkova, T. Y. Vadivasova, and V. S. Anishchenko, "Synchronization of chimera states in a network of many unidirectionally coupled layers of discrete maps," Regul. Chaotic Dyn. 23(7-8), 948-960 (2018).

${ }^{80}$ E. V. Rybalova, T. E. Vadivasova, G. I. Strelkova, V. S. Anishchenko, and A. S. Zakharova, "Forced synchronization of a multilayer heterogeneous network of chaotic maps in the chimera state mode," Chaos 29, 033134 (2019).

${ }^{81}$ I. Leyva, I. Sendiña-Nadal, J. A. Almendral, A. Navas, S. Olmi, and S. Boccaletti, "Explosive synchronization in weighted complex networks," Phys. Rev. E 88, 042808 (2013).

${ }^{82} \mathrm{X}$. Zhang, S. Boccaletti, S. Guan, and Z. Liu, "Explosive synchronization in adaptive and multilayer networks," Phys. Rev. Lett. 114, 038701 (2015).

${ }^{83}$ S. Jalan, V. Rathore, A. D. Kachhvah, and A. Yadav, "Inhibition-induced explosive synchronization in multiplex networks," Phys. Rev. E 99, 062305 (2019).

${ }^{84}$ Z. Ma, G. Zhang, Y. Wang, and Z. Liu, "Cluster synchronization in star-like complex networks,” J. Phys. A: Math. Theor. 41(15), 155101 (2008).

${ }^{85} \mathrm{P}$. V. Kuptsov and A. V. Kuptsova, "Variety of regimes of starlike networks of Henon maps," Phys. Rev. E 92, 042912 (2015).

${ }^{86} \mathrm{~J}$. Wang and Y. Zhang, "Network synchronization in a population of starcoupled fractional nonlinear oscillators," Phys. Lett. A 374(13-14), 1464-1468 (2010).

${ }^{87}$ A. Bergner, M. Frasca, G. Sciuto, A. Buscarino, E. J. Ngamga, L. Fortuna, and J. Kurths, "Remote synchronization in star networks," Phys. Rev. E 85, 026208 (2012).

${ }^{88}$ I. Fischer, R. Vicente, J. M. Buldú, M. Peil, C. R. Mirasso, M. C. Torrent, and J. García-Ojalvo, "Zero-lag long-range synchronization via dynamical relaying," Phys. Rev. Lett. 97, 123902 (2006).

${ }^{89}$ R. Gutiérrez, R. Sevilla-Escoboza, P. Piedrahita, C. Finke, U. Feudel, J. M. Buldú, G. Huerta-Cuellar, R. Jaimes-Reátegui, Y. Moreno, and S. Boccaletti, "Generalized synchronization in relay systems with instantaneous coupling," Phys. Rev. E 88, 052908 (2013).

${ }^{90} \mathrm{P}$. V. Kuptsov and A. V. Kuptsova, "Radial and circular synchronization clusters in extended starlike network of van der Pol oscillators," Commun. Nonlinear Sci. Numer. Simulat. 50, 115-127 (2017).

${ }^{91}$ L. V. Gambuzza, A. Cardillo, A. Fiasconaro, L. F. J. Gomez-Gardenes, and M. Frasca, "Analysis of remote synchronization in complex networks," Chaos 23, 043103 (2013).

${ }^{92}$ R. Banerjee, D. Ghosh, E. Padmanaban, R. Ramaswamy, L. M. Pecora, and S. K. Dana, "Enhancing synchrony in chaotic oscillators by dynamic relaying," Phys. Rev. E 85, 027201 (2012).

${ }^{93}$ L. V. Gambuzza, M. Frasca, L. Fortuna, and S. Boccaletti, "Inhomogeneity induces relay synchronization in complex networks,” Phys. Rev. E 93, 042203 (2016).

${ }^{94} \mathrm{R}$. Lozi, "Un attracteur entrahge du type attracteur de Henon," J. Phys. 39(C5), 9-10 (1978).

${ }^{95}$ Y. B. Pesin, "Dynamical systems with generalized hyperbolic attractors: Hyperbolic, ergodic and topological properties," Ergodic Theor. Dyn. Syst. 12, 123-151 (1992).

${ }^{96} \mathrm{M}$. Hénon, "Numerical study of quadratic area-preserving mappings," Quart. Appl. Math. 27, 291 (1969).

${ }^{97}$ V. S. Afraimovich and L. P. Shil'nikov, "Strange attractors and quasiattractors," in Nonlinear Dynamics and Turbulence, Interaction of Mechanics and Mathematics Series, edited by G. I. Barenblatt, G. Iooss, and D. D. Joseph (Pitman, Boston, MA, 1983), pp. 1-34. 